\title{
Safety and efficacy of oral fexofenadine in children with seasonal allergic rhinitis - a pooled analysis of three studies
}

Meltzer EO, Scheinmann P, Rosado Pinto JE, Bachert C, Hedlin G, Wahn U, Finn Jr AF, Ruuth E. Safety and efficacy of oral fexofenadine in children with seasonal allergic rhinitis - a pooled analysis of three studies.

Pediatr Allergy Immunol 2004: 15: 253-260. @ 2004 Blackwell Munksgaard

Allergic rhinitis is one of the most common clinical conditions in children; however, data regarding the safety of antihistamines in children with seasonal allergic rhinitis are limiting. To evaluate the safety and efficacy of fexofenadine in children with seasonal allergic rhinitis, data were pooled from three, double-blind, randomized, placebo-controlled, parallel-group, 2-week trials in children (6-11 year) with seasonal allergic rhinitis. All studies assessed fexofenadine $\mathrm{HCl} 30 \mathrm{mg}$ b.i.d.; two studies included fexofenadine $\mathrm{HCl}$ at 15 and $60 \mathrm{mg}$ b.i.d. Patients (and investigators) reported any adverse events during the trial. Physical examinations, including measurements of vital signs and laboratory tests, were performed. Efficacy assessments (total symptom score and individual symptom scores) were evaluated. Exposure to fexofenadine $\mathrm{HCl} 30 \mathrm{mg}$ b.i.d. and to any fexofenadine dose exceeded 10,000 and 17,000 patient days, respectively. Incidences of adverse events, and discontinuations because of adverse events, were low and similar across treatment groups. In the placebo group, $24.4 \%$ of subjects reported adverse events compared with $24.1 \%$ for fexofenadine $\mathrm{HCl} 30 \mathrm{mg}$ b.i.d., and $28.4 \%$ for all fexofenadine-treated groups. The most common adverse event overall was headache $(4.3 \%$ placebo; $5.8 \%$ fexofenadine $\mathrm{HCl} 30 \mathrm{mg}$ b.i.d.; and 7.2\% any fexofenadine doses). Treatment-related adverse events were similar across treatment groups with no sedative effects. Fexofenadine $\mathrm{HCl} 30 \mathrm{mg}$ b.i.d. was significantly superior to placebo in reducing the total symptom score and all individual seasonal allergic rhinitis symptoms, including nasal congestion $(\mathrm{p}<0.05)$.

Fexofenadine, at doses of up to $60 \mathrm{mg}$ b.i.d., is safe and non-sedating, and fexofenadine $\mathrm{HCl} 30 \mathrm{mg}$ b.i.d. effectively reduces all seasonal allergic rhinitis symptoms in children aged 6-11 years.

\author{
Eli O. Meltzer', Pierre Scheinmann², \\ Jose E. Rosado Pinto ${ }^{3}$, Claus Bachert ${ }^{4}$, \\ Gunilla Hedlin ${ }^{5}$, Ulrich Wahn ${ }^{6}$, Albert \\ F. Finn $\mathrm{Jr}^{7}$ and Erik Ruuth \\ ${ }^{1}$ Allergy and Asthma Medical Group and Research \\ Center, San Diego, CA, USA, ${ }^{2}$ Groupe Hospitalier \\ Necker-Enfants Malades, Paris, France, ${ }^{3}$ Department \\ of Allergology and Clinical Immunology, Hospital \\ Dona Estefânia, Lisbon, Portugal, ${ }^{4}$ Ghent University, \\ Ghent, Belgium, ${ }^{5}$ Department of Pediatrics, \\ Karolinska Institutet, Stockholm, Sweden, \\ ${ }^{6}$ Department of Pediatric and Pneumology and \\ Immunology, University Children's Hospital, Berlin, \\ Germany, ${ }^{7}$ National Allergy, Asthma and Urticaria \\ Centers, University Boulevard, Charleston, SC, USA, \\ ${ }^{8}$ Aventis, Paris, France
}

Key words: children; efficacy; fexofenadine; pooled analysis; safety; seasonal allergic rhinitis; worldwide

Professor Eli 0. Meltzer, Allergy and Asthma Medical Group and Research Center, 9610 Granite Ridge Drive, San Diego, CA, USA

Tel.: +1 8582682368

Fax: +1 8582685147

E-mail: eomeltzer@aol.com

Accepted 23 January 2004
Allergic rhinitis is one of the most common chronic conditions in childhood (1). In particular, many children who develop allergies to inhalant allergens in early life are at an increased risk of developing seasonal allergic rhinitis (SAR) and asthma in later childhood. This chronological progression of clinical manifestations of allergy is termed 'the allergic march'. Besides the potential serious implications of leading to asthma and other symptoms, children with untreated allergy symptoms have been shown to exhibit poorer learning ability compared with non-allergic children (2). Moreover, data indicate that almost a million school days are missed per year as a direct result of allergic rhinitis in the USA (3). Estimates of its prevalence in children, which vary from country to country, have been reported to be as high as $40 \%$ 
(4), and it appears that the incidence of allergic rhinitis in this patient population is rising (5).

Since 1942, antihistamines have been used as first-line treatment for SAR in patients of all ages (6-8). However, results from studies using firstgeneration antihistamines have demonstrated significant adverse effects as a result of their sedative effects, resulting in impairment of psychomotor and academic function in children (2). Fexofenadine (Allegra ${ }^{\circledR} /$ Telfast $^{\circledR}$, Aventis Pharmaceuticals, Kansas City, MO, USA), an optimal second generation antihistamine, demonstrates greater selectivity for the $\mathrm{H}_{1}$-receptor and lacks the anti-cholinergic and sedating adverse effects associated with many of the older antihistamines (9). Fexofenadine has been approved for the treatment of SAR and chronic idiopathic urticaria in adults, and large, randomized trials have demonstrated the efficacy and safety of this agent in adult populations (10-14). Furthermore, results of dose-response trials in healthy adults show that doses of up to $690 \mathrm{mg}$ b.i.d. administered for 1 month are safe and well-tolerated $(15,16)$. However, despite the greater emphasis on safety and tolerability in this population, data regarding the safety of antihistamines in children in controlled studies are limited. A previous report by Graft et al. (17) showed that doses of fexofenadine $\mathrm{HCl}$, up to $60 \mathrm{mg}$ b.i.d., are safe and well-tolerated in children aged 6-11 years, with no dose-related trends. In addition, a recent, worldwide study reported the efficacy and safety of fexofenadine $\mathrm{HCl}$ (30 $\mathrm{mg}$ b.i.d.) in children aged 6-11 years with SAR (18).

In order to further evaluate the safety of the recommended fexofenadine dose in this population, the safety results of these studies have been pooled. In addition, the efficacy of fexofenadine $30 \mathrm{mg}$ b.i.d. in reducing the symptoms of SAR was determined.

\section{Methods}

Patients

Three trials (studies I-III) were included in the pooled safety analysis. Children aged 611 years, with spring or fall SAR, and a history of SAR of approximately 1 year or more during at least one previous relevant season, were enrolled. Subjects were required to have a positive skin-prick test (wheal diameter $\geq 3 \mathrm{~mm}$ than that with diluent within 15 min of the skin prick) to at least one allergen for the current season. To facilitate objective characterization of the subject's allergies, patients in study I also required the presence of an allergen-specific immunoglobulin $\mathrm{E}$ ( $\mathrm{IgE}$ ) that was positive in the skin-prick test.

Excluded patients were (but were not limited to) those with a respiratory tract nasal infection, sinusitis, or otitis media within 30 days of study entry; clinically significant cardiovascular, hepatic, neurologic, psychiatric, endocrine or other major systemic disease; or immunotherapy treatment.

Written informed consent from a parent/ guardian and each child was obtained before inclusion in the study.

\section{Study design}

Studies I-III were double-blind, randomized, placebo-controlled, parallel-group, 2-wk trials. All three studies had a similar design with regard to patient selection criteria and, primary and secondary outcome measures. Study I was conducted at 148 centers in 15 countries: Argentina (16), Australia (nine), Austria (one), Chile (three), Finland (three), France (12), Germany (five), Israel (three), Italy (seven), Poland (10), Portugal (two), South Africa (18), Spain (six), Uruguay (two) and the USA (51) (18). Studies II and III were identical trials conducted at 58 investigative sites in the USA and Canada (17).

All three trials had a similar study design. At visit 1 (baseline), the children (with the help of their caregiver) provided a 12-hour reflective assessment of the severity of their allergy symptoms. Seasonal allergic rhinitis symptom scores were recorded for sneezing; rhinorrhea; itchy nose, palate, throat and/or ears; itchy, watery and/or red eyes; and nasal congestion. Each symptom was evaluated on a five-point scale: $0=$ absent; $1=$ mild; $2=$ moderate; $3=$ severe; $4=$ very severe. The total symptom score (TSS) was calculated by adding the individual symptom scores, excluding nasal congestion (maximum possible TSS $=16$ ). For inclusion at visit 1 , a reflective TSS $=6$, with two or more symptoms with a minimum score of 2 (moderate), was necessary. Following visit 1 , the studies included four additional visits: visit 2, randomization (day 1); visit 3, during double-blind treatment (day 610); visit 4, end of double-blind treatment (day 15-17); and visit 5, follow-up (day 22-24).

Following completion of a single-blind, placebo lead-in period, the children in study I were required to have a TSS $=5$ for the average of the last two 19:00-hours reflective TSS, and patients in studies II and III required a TSS $=5$ for $>60 \%$ of the placebo lead-in period to qualify for randomization to the double-blind phase of 
the study. All children were randomized to receive either fexofenadine $\mathrm{HCl} 30 \mathrm{mg}$ b.i.d. or matching placebo; studies II and III also included doses of 15 and $60 \mathrm{mg}$ b.i.d.. For the purpose of the present analysis, children treated with fexofenadine were analyzed in two groups: children treated with fexofenadine $30 \mathrm{mg}$ b.i.d. and children treated with all fexofenadine doses (15, 30 and $60 \mathrm{mg}$ b.i.d.). Safety data corresponding to the patient groups treated with either 15 or $60 \mathrm{mg}$ b.i.d. fexofenadine $\mathrm{HCl}$ are reported elsewhere (17). Tablets were taken at 19:00 and 7:00 hours ( \pm 1 hour) for 2 weeks, with no dosing instructions regarding food intake. Throughout the study, SAR symptoms were assessed daily at 19:00 $( \pm 1)$ hours for the previous 12-hour period (hereafter referred to as PM reflective) by the subject and caregiver, immediately prior to dosing.

The individual study protocols were approved by the appropriate ethics committees.

\section{Safety assessments}

Children (and investigators) reported any adverse events (AEs) during the study. In order that AE coding was uniform across studies, data from studies II and III were re-coded using the Hoechst Adverse Reaction Terminology System (HARTS) dictionary. Comparison of the original safety data with the results following recoding using HARTS showed that this did not have an effect on the safety profile of fexofenadine, with the incidence of adverse events and their severity remaining the same (data not shown). The AE profiles were pooled and presented for the following treatment groups: fexofenadine $\mathrm{HCl}$ $30 \mathrm{mg}$ b.i.d., all fexofenadine-treated subjects and placebo. Physical examinations, including vital signs measurements and laboratory tests, were performed in all three studies. Laboratory testing was performed on blood samples taken at visit 1 (screening) and visit 4 (end of treatment) and included assessments of liver function [alkaline phosphatase, total bilirubin, lactate dehydrogenase $(\mathrm{LDH})]$, renal function (creatinine), electrolytes (potassium), other chemistry (glucose, triglycerides) and hematology/hemoglobin (hematocrit, platelet count, white blood cell count). Study I assessed predefined change abnormal (PCA) and clinically noteworthy abnormal laboratory values (CNALVs), whereas studies II and III assessed outlier values; however, the values for the ranges of the CNALV and outliers were similar. In addition, the use of concomitant medications was also evaluated during the study. Studies II and III also recorded results from pre- and post-treatment electrocardiograms. These are reported elsewhere (17).

\section{Efficacy measurements}

Efficacy of fexofenadine was assessed only in those children treated with fexofenadine $30 \mathrm{mg}$ b.i.d. In all three studies, the primary outcome measures were mean change from baseline in the average PM reflective TSS throughout the doubleblind treatment period and the PM reflective assessment of individual symptom scores. The only difference in the studies is the definition of baseline TSS. For study I, baseline corresponded to the average assessment over the last 3 days prior to the first day of study medication; for studies II and III, baseline corresponded to the average over the whole placebo lead-in period. To assess the impact of this difference, the baseline for studies II and III was derived using the average TSS for the last 3 days prior to study medication. There is a slight decrease in the mean baseline for the placebo group and no change for the fexofenadine $\mathrm{HCl} 30 \mathrm{mg}$ group, indicating that the alternative methods of calculating baseline values did not preclude pooling of the three studies.

\section{Statistical analysis}

Children included in the safety evaluations were required to have been exposed to at least one dose of the double-blind treatment. Adverse events recorded by these children were descriptively summarized and no formal statistical comparisons were made. The efficacy analysis was undertaken on the modified intention-totreat (mITT) population, which was defined as all randomized subjects who received at least one dose of double-blind treatment and had a baseline and at least one double-blind period PM reflective assessment. Analysis of covariance (ANCOVA) was used to compare the efficacy of fexofenadine $\mathrm{HCl} 30 \mathrm{mg}$ b.i.d. with placebo, and baseline score was included as a continuous covariate, with treatment and pool center as fixed effects. A two-sided $95 \%$ confidence interval of the treatment difference was derived using adjusted least squares mean from the ANCOVA.

\section{Results}

Patient population and demographics

A total of 1810 children were included in the overall pediatric safety population (placebo, $\mathrm{n}=700$; fexofenadine $\mathrm{n}=1110$ ). Of these, 673 
children received fexofenadine $\mathrm{HCl} 30 \mathrm{mg}$ b.i.d. and 437 children received fexofenadine at doses of either $15 \mathrm{mg}$ or $60 \mathrm{mg}$ b.i.d. Exposure to fexofenadine $\mathrm{HCl} 30 \mathrm{mg}$ b.i.d. and to any fexofenadine dose exceeded 10,000 and 17,000 patient days, respectively.

The safety populations of the pooled studies were similar in terms of demographic characteristics (Table 1). For children receiving placebo or fexofenadine $\mathrm{HCl} 30 \mathrm{mg}$ b.i.d., $62.2 \%$ of the children were male and the mean age was 8.9 years.

As part of this pooled analysis, efficacy measurements were also determined. A total of 1804 pediatric subjects were evaluable for efficacy and included in the mITT population (671 children received fexofenadine $\mathrm{HCl} 30 \mathrm{mg}$ b.i.d.; 698 children received placebo). There were no marked differences in the demographic data of the mITT population either between studies or treatment groups. Similarly, within each study, there was no significant difference in baseline symptom scores between treatment groups.

\section{Safety assessments}

Adverse events. Incidences of AEs, including discontinuations because of AEs, were low and comparable across treatment groups in the pooled analysis (Table 2). In the placebo group, $24.4 \%$ (171 of 700 ) of subjects reported AEs compared with $24.1 \%$ (162 of 673) for fexofenadine $\mathrm{HCl} 30 \mathrm{mg}$ b.i.d., and $28.4 \%$ (315 of 1110 ) for all fexofenadine-treated $(15 \mathrm{mg}, 30 \mathrm{mg}$ and $60 \mathrm{mg}$ b.i.d.) children. The most common AE overall was headache, which occurred in $4.3 \%$ of placebo-treated children, $5.8 \%$ of fexofenadine $\mathrm{HCl} 30 \mathrm{mg}$ b.i.d.-treated children and in $7.2 \%$ of children treated with either 15,30 or $60 \mathrm{mg}$ b.i.d. fexofenadine.

AEs resulted in the withdrawal of six children receiving fexofenadine $\mathrm{HCl} 30 \mathrm{mg}$ b.i.d., one child receiving fexofenadine $\mathrm{HCl} 15 \mathrm{mg}$ b.i.d., one child receiving fexofenadine $\mathrm{HCl} 60 \mathrm{mg}$ b.i.d. and five children receiving placebo. The most common AE leading to discontinuation was asthma, which occurred in five children (three receiving placebo and two receiving fexofenadine $\mathrm{HCl} 30 \mathrm{mg}$ b.i.d.). No $\mathrm{AE}$ that resulted in discontinuation was attributed to study medication.

The number of treatment-related AEs (possibly related, probably related or definitely related to study treatment) was also comparable between treatment groups (Table 3). In the placebo group, a total of $3.4 \%$ (24 of 700 ) of children reported at least one treatment-related AE com-

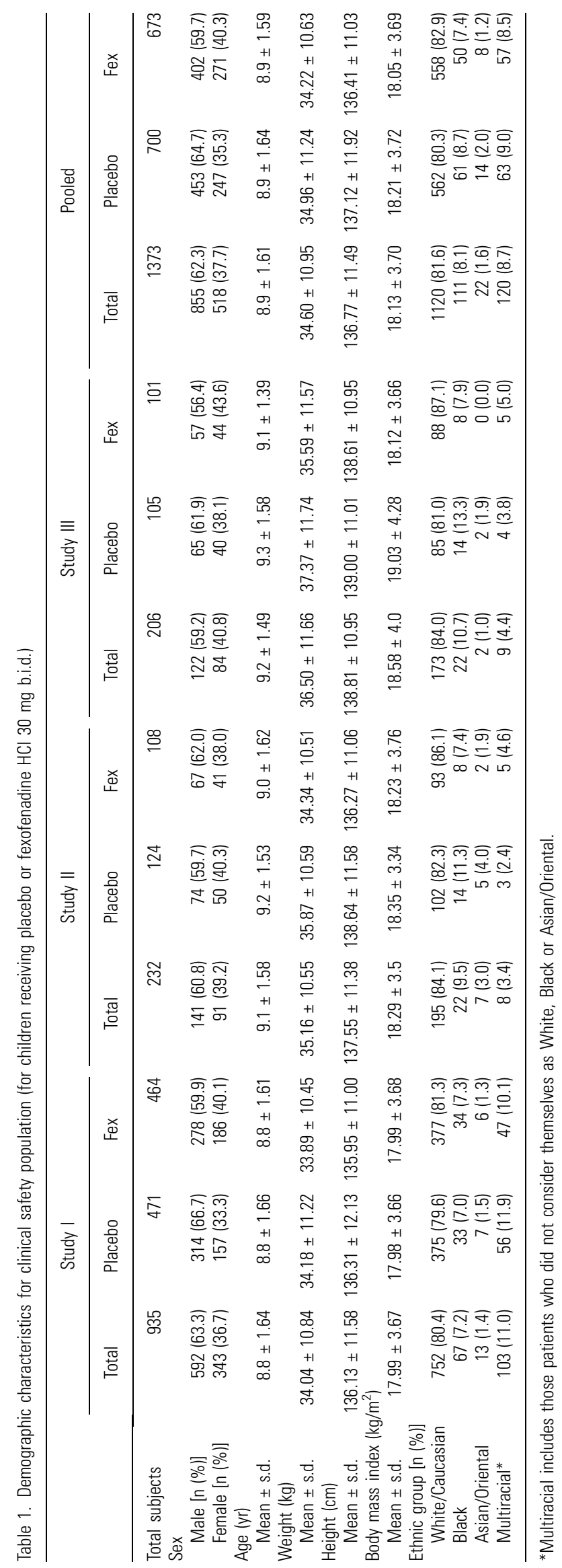


Table 2. Most frequently reported adverse events $(\geq 1 \%)$ - clinical safety population

\begin{tabular}{|c|c|c|c|}
\hline & \multicolumn{3}{|c|}{ Pooled data [\% (n)] } \\
\hline & Placebo & $\begin{array}{l}\text { Fexofenadine } \\
30 \mathrm{mg} \text { b.i.d. }\end{array}$ & $\begin{array}{l}\text { Fexofenadine } \\
\text { all }^{*}\end{array}$ \\
\hline $\begin{array}{l}\text { Subjects evaluable for safety } \\
\text { in double-blind period }\end{array}$ & $100(700)$ & $100(673)$ & $100(1110)$ \\
\hline Subjects with AEs & 24.4 (171) & $24.1(162)$ & $28.4(315)$ \\
\hline \multicolumn{4}{|l|}{ Coded Term } \\
\hline Headache & $4.3(30)$ & $5.8(39)$ & $7.2(80)$ \\
\hline Upper respiratory infection & $1.3(9)$ & $2.1(14)$ & $2.2(24)$ \\
\hline Cough increased & $0.9(6)$ & $1.6(11)$ & $1.7(19)$ \\
\hline Epistaxis & $1.1(8)$ & $1.5(10)$ & $1.5(17)$ \\
\hline Accidental injury & $1.6(11)$ & $1.3(9)$ & $1.6(18)$ \\
\hline Sore throat & $1.6(11)$ & $1.2(8)$ & $1.7(19)$ \\
\hline Rash & $0.6(4)$ & $1.2(8)$ & $1.2(13)$ \\
\hline Nausea & $0.1(1)$ & $1.2(8)$ & $1.0(11)$ \\
\hline Fever & $0.4(3)$ & $1.0(7)$ & $1.3(14)$ \\
\hline Sinusitis & $0.9(6)$ & $1.0(7)$ & $0.8(9)$ \\
\hline Otitis media & $0.1(1)$ & $1.0(7)$ & $0.8(9)$ \\
\hline Pain & $0.3(2)$ & $0.9(6)$ & $1.1(12)$ \\
\hline Respiratory disorder & $0.7(5)$ & $0.9(6)$ & $0.9(10)$ \\
\hline Pharyngitis & $0.4(3)$ & $0.9(6)$ & $0.9(10)$ \\
\hline Gastrointestinal pain & $1.9(13)$ & $0.7(5)$ & $1.4(15)$ \\
\hline Vomiting & $0.4(3)$ & $0.7(5)$ & $0.8(9)$ \\
\hline Dyspepsia & $0.1(1)$ & $0.7(5)$ & $0.7(8)$ \\
\hline Asthma & $2.0(14)$ & $0.6(4)$ & $0.5(5)$ \\
\hline Infection & $1.9(13)$ & $0.4(3)$ & $1.4(16)$ \\
\hline Flu syndrome & $0.3(2)$ & $0.4(3)$ & $0.6(7)$ \\
\hline Ear pain & $0.7(5)$ & $0.4(3)$ & $0.5(5)$ \\
\hline Chest pain & 0 & $0.3(2)$ & $0.5(5)$ \\
\hline Diarrhea & $0.1(1)$ & $0.3(2)$ & $0.4(4)$ \\
\hline
\end{tabular}

${ }^{*}$ Fexofenadine $\mathrm{HCl}$ at doses of 15,30 and $60 \mathrm{mg}$ b.i.d.

$\mathrm{AE}$, adverse event.

Table 3. Most frequently reported $(>0.5 \%)$ treatment-related adverse events clinical safety population

\begin{tabular}{|c|c|c|c|}
\hline & \multicolumn{3}{|c|}{ Pooled data } \\
\hline & Placebo & $\begin{array}{l}\text { Fexofenadine } \\
30 \mathrm{mg} \text { b.i.d. }\end{array}$ & $\begin{array}{l}\text { Fexofenadine } \\
\text { All }^{*}\end{array}$ \\
\hline $\begin{array}{l}\text { Subjects evaluable for safety } \\
\text { in double-blind period, \% (N) }\end{array}$ & $100(700)$ & $100(673)$ & $100(1110)$ \\
\hline $\begin{array}{l}\text { Subjects with possibly related } \\
\text { AEs, \% (N) }\end{array}$ & $3.4(24)$ & $4.0(27)$ & $5.2(58)$ \\
\hline \multicolumn{4}{|l|}{ Coded Term } \\
\hline Headache & $1.0(7)$ & $1.0(7)$ & $1.4(15)$ \\
\hline Gastrointestinal pain & $0.6(4)$ & $0.1(1)$ & $0.4(4)$ \\
\hline Rash & $0.1(1)$ & $0.3(2)$ & $0.5(5)$ \\
\hline Insomnia & 0 & $0.1(1)$ & $0.4(4)$ \\
\hline Somnolence & $0.4(3)$ & $0.1(1)$ & $0.1(1)$ \\
\hline
\end{tabular}

${ }^{*}$ Fexofenadine $\mathrm{HCl}$ at doses of 15,30 and $60 \mathrm{mg}$ b.i.d. $\mathrm{AE}$, adverse event.

pared with $4.0 \%$ (27 of 673 ) for fexofenadine $\mathrm{HCl} 30 \mathrm{mg}$ b.i.d., and $5.2 \%$ (58 of 1110 ) for all fexofenadine treatment groups. No sedative effect or other class effects evident with firstgeneration antihistamines were observed. The most commonly reported treatment-related $\mathrm{AE}$ was headache, which had an incidence of $1.0 \%$ (7 of 700 ) for placebo, $1.0 \%$ (7 of 673 ) for fexofenadine $\mathrm{HCl} 30 \mathrm{mg}$ b.i.d., and 1.4\% (15 of $1110)$ for all fexofenadine-treated groups. The majority of the treatment-related AEs were considered to be of mild or moderate intensity.

The number of children requiring concomitant medication (i.e. medication that was not provided in the study protocol) was comparable between treatment groups. For study I, the percentage of children taking concomitant medication was $10.8 \%$ (51 of 471 ) for placebo and $10.3 \%$ (48 of 464 ) for those children treated with fexofenadine $\mathrm{HCl} 30 \mathrm{mg}$. For studies II and III, the percentage of children taking concomitant medication was $28.8 \%$ (66 of 229) and $24.9 \%$ (161 of 646) for placebo- and fexofenadinetreated children, respectively.

Two subjects in the three studies reported serious AEs. One child in study I experienced neutropenia in the fexofenadine-treated group (30 $\mathrm{mg}$ b.i.d.) on routine evaluation that was thought to be related to treatment by the investigator. Laboratory tests revealed decreased neutrophil levels on day 15 following 14 days of double-blind treatment $\left(0.4 \times 10^{9} / 1\right.$ compared with $2.3 \times 10^{9} / 1$ at screening). This event was reported as mild in intensity and the child was asymptomatic, and recovered without sequelae. Total leukocyte counts for this child remained stable during follow-up, but still below the normal range. Because serologic work was consistent with recent seroconversions for Mycoplasma pneumoniae and Varicella IgG, subclinical infections may offer an alternative explanation for the event. In addition, in study III, one child in the $30 \mathrm{mg}$ b.i.d. fexofenadine-treated group experienced increased asthma and was diagnosed with status asthmaticus. The investigator felt that the event was severe, and unlikely to be related to study medication, and instead related to underlying disease with exposure to outdoor allergens as a trigger.

Vital signs and clinical laboratory evaluations. No clinically relevant changes were observed in vital signs data between fexofenadine and placebotreated groups.

With the exception of one child with neutropenia described above, in all studies and in all treatment groups there were no clinically relevant differences in laboratory values. In study I, the most frequently observed PCA and CNALV for both treatment groups was an increase in eosinophils. In studies II and III, the most frequent outlier was high hematocrit levels. 


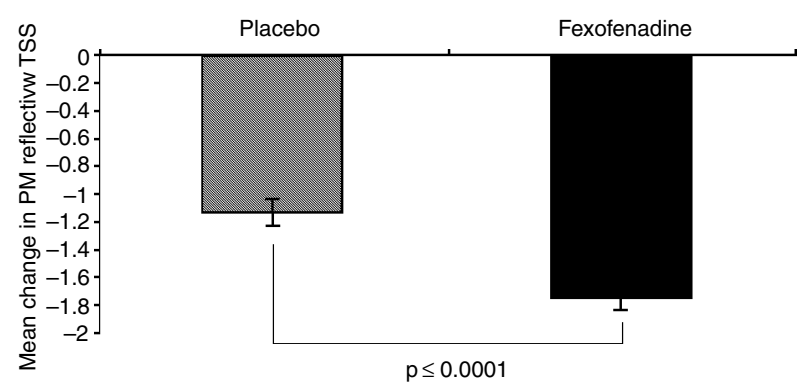

Fig. 1. Mean ( \pm s.e.) change in PM reflective TSS with mITT population; $\mathrm{p} \leq 0.0001$ vs. placebo (for children receiving placebo or fexofenadine $\mathrm{HCl} 30 \mathrm{mg}$ b.i.d.).

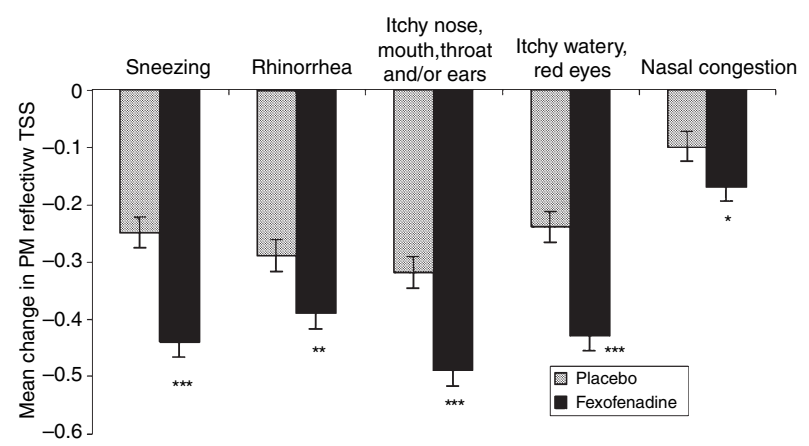

Fig. 2. Reflective individual symptom scores with mITT population. $* \mathrm{p}<0.05, * * \mathrm{p}<0.01, * * * \mathrm{p} \leq 0.0001$ vs. placebo (for children receiving placebo or fexofenadine $\mathrm{HCl}$ $30 \mathrm{mg}$ b.i.d.).

\section{Efficacy assessments}

Efficacy analysis showed that fexofenadine $\mathrm{HCl}$ $30 \mathrm{mg}$ b.i.d. was significantly superior to placebo in the PM reflective TSS pooled analysis $(\mathrm{p} \leq 0.0001 ;$ Fig. 1$)$. The mean change from baseline was -1.75 for the fexofenadine-treated group and -1.14 for the placebo group.

In the pooled analysis, all PM reflective individual symptom scores, including nasal congestion, were significantly reduced compared with placebo (sneezing $\mathrm{p} \leq 0.0001$; rhinorrhea $\mathrm{p}<0.0058$; itchy nose, mouth, throat and/or ears $\mathrm{p} \leq$ 0.0001 ; itchy, watery, red eyes $\mathrm{p} \leq 0.0001$; nasal congestion $\mathrm{p}<0.0334$ ) (Fig. 2).

\section{Discussion}

The safety data from these three pooled studies showed that over a 2 -wk period fexofenadine $\mathrm{HCl}$ (30 mg b.i.d.) is well tolerated, with a placebo-like safety profile in children with SAR. The most commonly reported AE was headache, which occurred with a similar incidence in both fexofenadine- and placebo-treated groups. The results of this study also demonstrate that fexofenadine $\mathrm{HCl}$ (30 $\mathrm{mg}$ b.i.d.) is effective in reducing both the TSS and individual symptom scores of SAR compared with placebo.

The combined results reported here are consistent with the previously published safety data for fexofenadine studies II and III $(17,18)$, in which there were no dose-related trends for AEs in children. Similar to the present study, the report by Graft et al. (17) showed that headache was the most frequently reported $\mathrm{AE}$, with a similar incidence for all doses of fexofenadine (15, 30 and $60 \mathrm{mg}$ b.i.d.) compared with placebo. In addition, early investigation of the clinical pharmacology of fexofenadine in healthy children has shown single doses of 30 and $60 \mathrm{mg}$ are safe and well-tolerated (19). These findings are supported by the established safety profile of fexofenadine in adults for treatment of allergic rhinitis $(15,16)$. The two most commonly reported AEs in these long-term studies were headache and viral infection $(15,16)$. Moreover, since first approved for use in children in 1999, a systematic review of spontaneous adverse event reports has not revealed any new risk that could modify the current safety profile of fexofenadine tablets.

The potential for sedation is important in terms of safety, and the ability of both adults and school children to undertake everyday activities. Superior cognitive functions such as attention, memory, co-ordination, cognition and psychomotor performance may all be affected by sedation and central nervous system impairment, with associated safety implications. The findings from this study show that no clinically meaningful sedative or other class effects associated with first-generation antihistamines were observed. These findings are consistent with studies in adults, in which fexofenadine, even at doses in excess of those recommended by the manufacturer, did not impair cognitive or psychomotor function $(15,20,21)$. In particular, Hindmarch and Shamsi (21) conducted a comprehensive review of published data from well-controlled trials evaluating the effects of antihistamines on sedation, psychomotor performance and cognition in healthy volunteers, using standardized, quantitative methods determining drug-related effects. Unlike cetirizine, fexofenadine was not associated with objective or subjective performance or cognitive impairment in any test, even at higher than recommended doses (21). Furthermore, qualitative and quantitative cerebral $\mathrm{H}_{1}$ binding data from PET studies have shown that fexofenadine does not bind to the $\mathrm{H}_{1}$-receptor in the brain, suggesting that it does not cross the blood-brain barrier, thereby supporting the lack of somnolence or impairment observed with fexofenadine in clinical studies (22). 
A number of reports have linked some antihistamines to prolongation of the QT interval corrected for heart rate (QTc) interval when administered in the presence of hepatic impairment or with drugs that inhibit the cytochrome P450 hepatic enzyme system. Electrocardiograms were performed in studies II and III, and showed that fexofenadine has no effect on the QTc (17). Other studies, conducted in children and adults with SAR, in healthy children and adults, and in drug interaction studies with ketoconazole and erythromycin, also showed no effect of fexofenadine on the QTc compared with placebo $(15,17$, 20, 23). Furthermore, the cardiovascular safety of fexofenadine in adults has been established at daily doses up to $690 \mathrm{mg}$ b.i.d. (24).

Traditionally, antihistamines have not been considered to be effective in relieving nasal congestion (25-27) and, accordingly, secondgeneration antihistamines are not indicated for this purpose. The results of this pooled analysis shows that fexofenadine $\mathrm{HCl}(30 \mathrm{mg}$ b.i.d.) is effective in reducing both the TSS and all individual symptoms, including nasal congestion, in children with SAR. These results are consistent with the recently published efficacy data from one of the studies reported here, in which fexofenadine was shown to be efficacious across all symptoms of SAR (18). Similarly, other studies in adults with SAR have shown significant efficacy with fexofenadine in relieving all symptoms of SAR, including nasal congestion $(10,12,14)$. It is hypothesized that the ability of certain antihistamines, such as fexofenadine, to inhibit the late-phase response may be attributable in part to anti-inflammatory effects that are not necessarily directly related to $\mathrm{H}_{1}$-receptor antagonism (28-30).

\section{Conclusion}

The results from this pooled analysis of three clinical studies show that fexofenadine $\mathrm{HCl}$ at doses of 15,30 and $60 \mathrm{mg}$ b.i.d. is safe and well tolerated in children aged 6-11 years over a period of 2 weeks. In this large pediatric population, fexofenadine is also effective at relieving all symptoms of SAR, including nasal congestion.

\section{Acknowledgment}

This study was supported by Aventis Pharmaceuticals.

\section{References}

1. Simons FER. $\mathrm{H}_{1}$-antihistamines in children. In: Simons FER, ed. Histamine and H1-Antihistamines in Allergic Disease, 2nd edn. New York: Marcel Dekker, Inc 2002: 437-64.
2. Vuurman E, van Veggel L, Uiterwijk M, Leutner D, O'Hanlon JF. Seasonal allergic rhinitis and antihistamine effects on children's learning. Ann Allergy 1993: 71: 121-6.

3. Malone D, Lawson K, Smith D, Arrighi HM, BatTISTA C. A cost of illness study of allergic rhinitis in the United States. J Allergy Clin Immunol 1997: 99: 22-27.

4. The International Study of Asthma and Allergies in Childhood (ISAAC) Steering Committee. Worldwide variation in prevalence of symptoms of asthma, allergic rhinoconjunctivitis, and atopic eczema: ISAAC. Lancet 1998: 351: 1225-32.

5. Meltzer EO. An overview of current pharmacotherapy in perennial rhinitis. J Allergy Clin Immunol 1995: 95: 1097-110.

6. Druce HM, Kaliner MA. Allergic rhinitis. JAMA 1988: 259: 260-3.

7. Staord CT. Allergic rhinitis. A useful guide to diagnosis and treatment. Postgrad Med 1987: 81: 147-51, $54,57$.

8. Tarnasky PR, Van Arsdel PP. Antihistamine therapy in allergic rhinitis. J Fam Pract 1990: 30: 71-80.

9. Howarth PH. The choice of an $\mathrm{H}_{1}$-antihistamine for the 21st century. Clin Exp Allergy Rev 2002: 2: 18-25.

10. Howarth P, Stern M, Roi L, Reynolds R, Bousquet J. Double-blind, placebo-controlled study comparing the efficacy and safety of fexofenadine hydrochloride (120 mg and $180 \mathrm{mg}$ once-daily) and cetirizine in seasonal allergic rhinitis. J Allergy Clin Immunol 1999: 104: 927-33.

11. Finn A, Kaplan A, Fretwell R, Qu R, Long J. A double-blind, placebo-controlled trial of fexofenadine $\mathrm{HCl}$ in the treatment of chronic idiopathic urticaria. J Allergy Clin Immunol 1999: 104: 1071-8.

12. Casale TB, Andrade C, Qu R. Safety and efficacy of once-daily fexofenadine $\mathrm{HCl}$ in the treatment of autumn seasonal allergic rhinitis. Allergy Asthma Proc 1999: 20: 193-8.

13. Mosges R, van Cauwenberge P, The Star Study Investigating Group. Fexofenadine and loratadine exhibit rapid onset of relief, but only fexofenadine maintains efficacy over a 2 -week study period. Allergy 2000: 55(Suppl. 63): 281.

14. van Caumenberge P, Juniper EF. Comparison of the efficacy, safety and quality of life provided by fexofenadine hydrochloride $120 \mathrm{mg}$, loratadine $10 \mathrm{mg}$ and placebo administered once daily for the treatment of seasonal allergic rhinitis. Clin Exp Allergy 2000: 30: 891-9.

15. Mason J, Reynolds R, Rao N. The systematic safety of fexofenadine HCI. Clin Exp Allergy 1999: 29(Suppl 3): 163-70.

16. Nathan R, Mason J, Bernstein D, et al. The long-term safety and tolerability of fexofenadine in healthy subjects. Clin Drug Invest 1999: 18: 317-28.

17. Graft DF, Bernstein Di, Goldsobel A, Meltzer EO, Portnoy J, Long J. Safety of fexofenadine in children treated for seasonal allergic rhinitis. Ann Allergy Asthma Immunol 2001: 87: 22-26.

18. Wahn U, Meltzer EO, FInN AF, et al. Fexofenadine is efficacious and safe in children (aged 6-11 years) with seasonal allergic rhinitis. J Allergy Clin Immunol 2003: 111: 763-9.

19. Simons FER, Bergman JN, Watson WTA, Simons KJ. The clinical pharmacology of fexofenadine in children. J Allergy Clin Immunol 1996: 98: 1062-4. 


\section{Meltzer et al.}

20. Russell T, Stoltz M, Weir S. Pharmacokinetics, pharmacodynamics, and tolerance of single- and multiple-dose fexofenadine hydrochloride in healthy male volunteers. Clin Pharmacol Ther 1998: 64: 612-21.

21. Hindmarch I, Shamsi Z. Antihistamines: models to assess sedative properties, assessment of sedation, safety and other side-effects. Clin Exp Allergy 1999: 29(Suppl. 3): $133-42$.

22. Dogan A, Oord S, Catafau A, et al. Cerebral H1-receptor pattern at baseline and after a single oral dose of the antihistamine fexofenadine. Ann Allergy Asthma Immunol 2000: 86: 108.

23. Pratt C, Brown A, Rampe D, et al. Cardiovascular safety of fexofenadine HCl. Clin Exp Allergy 1999: 29(Suppl. 3): 212-6.

24. Pratt CM, Mason J, Russell T, Reynolds R, AhlbrandT R. Cardiovascular safety of fexofenadine HCl. Am J Cardiol 1999: 83: 1451-4.

25. Bousquet J, van Caunenberge P, Khaltaev N, Aria Workshop Group. Guidelines on allergic rhinitis and its impact on asthma (ARIA) in collaboration with the world health organisation (WHO). J Allergy Clin Immunol 2001: 108: S: 147-336.
26. Kemp JP, Buckley CE, Gershwin ME, et al. Multicenter, double-blind, placebo-controlled trial of terfenadine in seasonal allergic rhinitis and conjunctivitis. Ann Allergy 1985: 54: 502-9.

27. Clissold SP, Sorkin EM, GoA KL. Loratadine. A preliminary review of its pharmacodynamic properties and therapeutic efficacy. Drugs 1989: 37: 42-57.

28. Abdelaziz MM, Devalia JL, Khair OA, Bayram H, Prior AJ, DAvies RJ. Effect of fexofenadine on eosinophil-induced changes in epithelial permeability and cytokine release from nasal epithelial cells of patients with seasonal allergic rhinitis. J Allergy Clin Immunol 1998: 101: 410-20.

29. Marone G, Gentile M, Petraroli A, De Rosa N, Triggiani M. Histamine-induced activation of human lung macrophages. Int Arch Allergy Immunol 2001: 124: 249-52.

30. Paolieri F, Battifora M, Riccio A, et al. Terfenadine and fexofenadine reduce in vitro ICAM-1 expression on human continuous cell lines. Ann Allergy Asthma Immunol 1998: 81: 601-7. 